\title{
Glove-Based Virtual Interaction for the Rehabilitation of Hemiparesis Stroke Patient
}

\author{
Khairunizam Wan, Aswad A.R., Rashidah Suhaimi and Nazrul H. Adnan \\ Advanced Intelligent Computing and Sustainability Research \\ School of Mechatronic, Universiti Malaysia Perlis, Malaysia \\ khairunizam@unimap.edu.my \\ D. Hazry, Zuradzman M. Razlan and Shahriman A.B. \\ Centre of Excellence for Unmanned Aerial Systems (COEUAS) \\ Universiti Malaysia Perlis, Malaysia \\ Mohd Asri Ariffin and Haslina M \\ School of Health Science, \\ Kampus Kesihatan Universiti Sains Malaysia, Malaysia
}

\begin{abstract}
This paper proposes the rehabilitation of a stroke patient by using dataglove called GloveMAP. GloveMAP is a low cost hand glove developed by using the flexible bending sensor and an accelerometer attached at the position of the fingers and wrist, respectively. In the studies, 22 fundamental movements of arm are evaluated and the results are employed to design the virtual environment. The combination of these fundamental movements focuses on Hemiparesis sufferers which are the most common in the stroke and have the inability to move one side of the body. The experimental results show the preliminary studies to investigate muscle activities. The research outcomes are used to design the rehabilitation based virtual environment (VR).
\end{abstract}

Keywords: Dataglove, Virtual Reality (VR), rehabilitation, stroke.

\section{Introduction}

Strokes have been one of the major causes for long term disability. Death cases from stroke have decreased considerably but the survivors usually have to live with some form of disability. Given stroke cause damage to the brain by forming blood clot in the brain artery, the complexity of the brain may affect the stroke patient in various forms [1, 2, 3].

Virtual Reality (VR) is a digital environment which can be fully manipulated by the respective party. VR has its various function in today's development in medical area which most have been used by the doctor for surgery simulation and also for psychological therapy for the mind. As it stand VR has serve its purpose in the development of this project for rehabilitation of the stroke patient [4-7].
This paper proposes the impairment of the stroke patient to rehabilitate by using VR approach. The study includes the research in investigation of muscle activities where the rehabilitation takes place.

This research paper organized as subsequent; Section 2 encompasses literature review of the related research and approach toward VR based stroke therapy. Section 3 presents the methodologies of applied procedures. Section 4 is divided into 2 sections, first section states about experiment setup where second section demonstrates the results of experiments. Finally section 5 expresses the conclusions over current research.

\section{Literature review}

Worldwide stroke is the second leading cause of death, responsible for 4.4 million or equivalent to $9 \%$ of the total 50.5 million deaths each year, and only $10 \%$ recover almost completely while some other survivor 
have to live with few disability or impairment. Thus this stroke patient needs special care and rehabilitation to gain back their body function. Most people over 55 years of age and with a male gender of family history of stroke could be one of many vital factor risk of getting a stroke.

Nowadays exists various application of the VR which have make rise to the development of interfacing devices such as dataglove, head-mounted display (HMD) and mechanical suit [6,8]. The application has various usages in the development therapy for the disability patient such as stroke patient. In this modern age lots of equipment have been develop for this VR applications. Though this technique requires a high cost to develop thus a lot improvement can still be made for this VR application.

Therapy by using VR is widely used in the society by integrating perceptions and experience within a simulation for the phobic patient. Rehabilitation can be made easy for the stroke patient as VR can help in attaining portable rehab center by remotely control the virtual exercise for the patient. Furthermore VR creates an environment where all objects can be created digitally allowing the user or patient to interact with the virtual object by using external hardware.

In this research, the dataglove called GloveMAP is used by the stroke patient to interact with the VR environments [9-11]. The most challenging part will be the designation of the virtual object installed in the virtual environments. The consultations from the medical doctor and the stroke therapist are required in order to design VR based stroke rehabilitation environments.

\section{Research methodologies}

Fig. 1 shows the flow chart of the proposed methods. The proposed works are divided into 2 parts, which are the design of the Virtual Reality (VR) environments and the rehabilitation therapist [9]. Several preliminary experiments are conducted to design the VR environments. The purpose is to investigate the best sequence of arm movements for the rehabilitation purpose. In the rehabilitation process, the stroke patient has an option to choose the VR environments, which is suitable to their stage of the stroke.

\subsection{Hemiparesis Stroke}

Hemiparesis stroke attacks when blood flow to the brain stopped. The oxygen and blood that supply to the brain will stop which result in the damage of the brain which can affect the important body parts that control daily activities. Hemiparesis sufferer will experience one side of weakness. They often experience balance impairments which cause difficulties to perform movements such as grabbing objects, drinking from a glass, eating and using bathroom. The loss of abilities of movements due to stroke depends on the brain injury area.

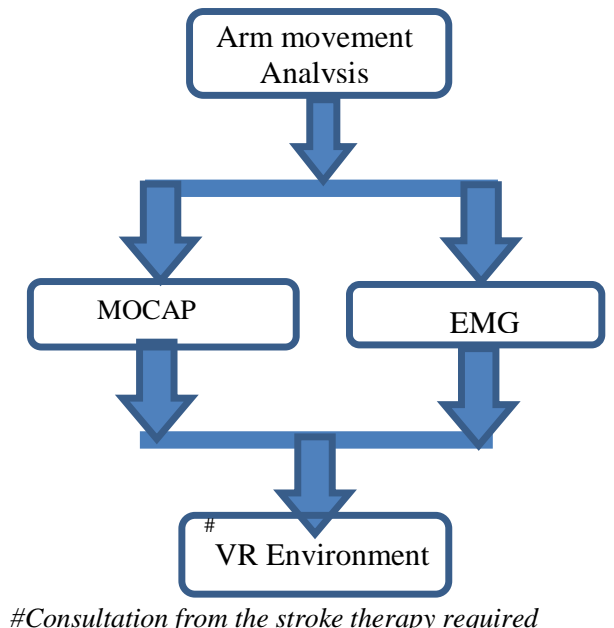

(a)

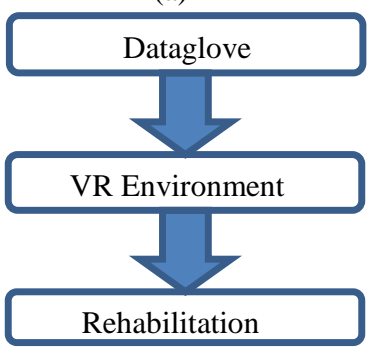

(b)

Fig.1. (a) Designing the VR environment (b) Rehabilitation therapy

\subsection{Fundamental Movements in Stroke Rehab}

\subsubsection{Rotational Angles of Human Arm}

Human arm consist of 7 DOF which are located at the shoulder, elbow and wrist. Shoulder part has 3 DOF for pitch, yaw and roll. While the elbow only has 1 DOF which make it can bend up and down. Finally the wrist that has 3 DOF for bending up and down, side to side 
deviation and twisting which make a total of 7 DOF for human arm [12].

Anatomical movements for hemiparesis stroke consist of several general movements that involve the shoulder, elbow, wrist and fingers. The movement carry out must be in correct range of motion to avoid injury during rehabilitation. Muscle is the tissues that provide movement for human body structure and the movement are classified by the direction of movement by the affected structures. Movements beyond the normal limit will be added with the prefix hyper-such as hyper flexion and hyper extension. Such movements are very important in medical field. General anatomical motion with its opposites includes flexion, extension, abduction, adduction, internal rotation, external rotation, elevation and depression

\subsection{Arm kinematics}

Flexion and extension are the motion of adjusting angle between two parts by bending or straightening the parts. While, abduction and adduction are adjusting relation to midline of the body by pulling the body structure away or toward the midline, the abduction and adduction of the wrist are called as radial and ulnar deviation. Internal and external rotations are rotating the body parts inwards or outwards. The movements of adjusting the elevation in superior or inferior direction are elevation and depression. Special motions for hands and feet are pronation and supination which are the rotation of the forearm.

Human arm structure shows the rotational angles of shoulder and elbow define as yaw, pitch and roll. 3 DOF at the shoulder define the pitch angle $\beta$ which is the rotation around $y$-axis for pronation and supination, yaw angle $\alpha$ which is the rotation around z-axis for abduction and adduction, while flexion and extension stand for roll angle $\gamma$ which rotate around x-axis. The DOF at the elbow must be considered as two which are the pitch and roll angle, however pronation and supination will not change the hand position. A single transformation matrix can be formed by calculating the combination of eulerian angles in the order of $\mathrm{Y}-\mathrm{X}-\mathrm{Y}$ and $\mathrm{Z}-\mathrm{X}-\mathrm{Z}$ and the angles can be computed.

\subsection{Virtual Reality (VR) software}

Since this project needs to do the virtual rehabilitation therapy, the proposed software is Quest3D which is a platform of the VR system. Quest3D is the software for developing real-time 3D Microsoft Windows applications. It's consists of only a few high level software tools and almost all tasks are performed identical to the hardware. By using the software development kit, users can build their own components for Quest3D and build support for specific hardware likes dataglove. The Virtual Reality Peripheral Network (VRPN) will be used to interface the dataglove with the VR environment and Head Mounted Display (HMD). VRPN is a zero cost, open source tool that can handle many VR devices.

\section{Experiments}

\subsection{Experimental setup}

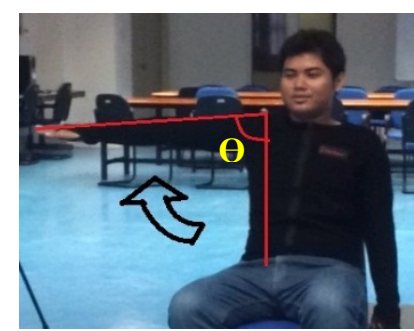

Fig. 2. The subject perform the fundamental movement

Table 1. The location of marker placement

\begin{tabular}{|c|l|c|}
\hline No. & \multicolumn{1}{|c|}{ Location } & ID Number \\
\hline 1 & Shoulder & P1 \\
\hline 2 & Elbow & P2 \\
\hline 3 & Wrist & P3 \\
\hline 4 & Trapezium & P4 \\
\hline 5 & Index & P5 \\
\hline 6 & Middle & P6 \\
\hline
\end{tabular}
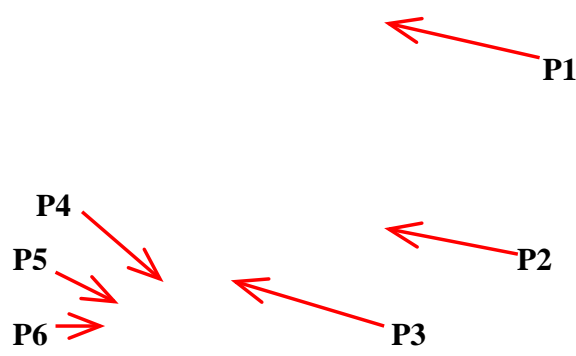

Fig. 3. Position of the 6 markers 
In the experiments, 1 subject was selected to perform the fundamental movements of the arm as shown in Fig. 2. The movements were captured by using the motion capture system Qualisys Track Manager (QTM) software. The QTM was calibrated each time in the experiment to reduce the tolerance of the 3D measurement. Six markers were attached to the right arm of the subject, which was at the location of shoulder, elbow, wrist, and fingers (Index and middle) as shown in Fig. 3.

\subsection{Experimental results}

\section{Shoulder Abduction}
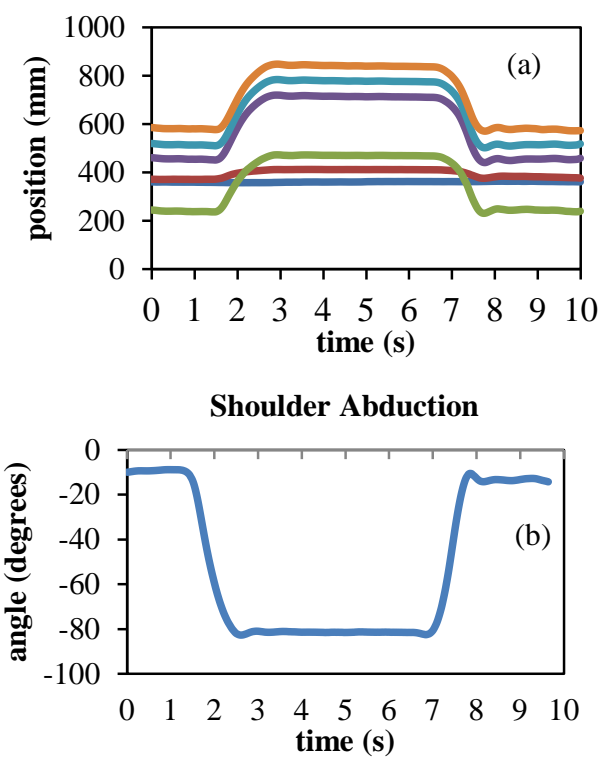

Fig. 4. Experimental results (a) Position for shoulder abduction (b) angle of shoulder abduction

Fig. 4(a) shows the markers at the position of shoulder (P1) and elbow (P2) experienced very little movement while the markers at the position of wrist (P3), index finger (P5) and middle finger (P6) show significant movement which about $250 \mathrm{~mm}$ of the vector magnitude.

\section{Conclusions}

The paper proposes the rehabilitation therapy of the stroke patient by interacting dataglove with the VR environments. Preliminary experiments are conducted to investigate the motion sequence of the arm by using MOCAP and EMG sensors for the purpose of designing the VR environments. The stroke patient does picks and place activities of the virtual objects in the VR environments. Based on these preliminary results, the portable stroke rehabilitation therapy device could be developed in the future. As the future work, the functional movements of the arm will be further investigated.

\section{Acknowledgements}

This work is supported by the MOSTI Science Fund Research Grant (MOSTI: 9005-00059) awarded by the Ministry of Education, Malaysia and Innovation to UniMAP.

\section{References}

1. Engelter ST, Amort M, Jax F et al., Optimizing the risk estimation after a transient ischaemic attack-the ABCDE? Score, Eur. J. Neurol, 19: pp. 55-61.

2. Mendis S, Prevention and care of stroke in low- and middle-income countries; the need for a public health perspective, Int. J. Stroke, 5: pp. 86-91.

3. Visintin M, Barbeau H, Korner-Bitensky N, Mayo NE, A new approach to retrain gait in stroke patients through body weight support and treadmill stimulation, Stroke, 19:pp. 1122-1128.

4. Holden M, Virtual Environments for Motor Rehabilitation: Review. CyberPsychology and Behavior 8(3), pp. 187-211.

5. Forducey P. et al., Tele-Rehabilitation using the Rutgers Master II glove following Carpal Tunnel Release surgery. IEEE Trans Neural Syst Rehabil Eng., 15(1): 43-49.

6. Deutsch J., Latonio J. and Burdea G, Rehabilitation of Musculoskeletal Injury Using the Rutgers Ankle Haptic Interface: Three Case Reports. Eurohaptics'01, pp. 1116.

7. Sveistrup H., Mccomas J. and Thornton M., Experimental Studies of Virtual Reality-Delivered Compared to Conventional Exercise Programs for Rehabilitation. CyberPsychology \& Behavior, 6(3): pp. 245-249.

8. Rose FD et al., Experimental Studies of Virtual RealityDelivered Compared to Conventional Exercise Programs for Rehabilitati Virtual Reality: an Assistive Technology in Neurological Rehabilitation. Curr. Opin. Neurol., 9(6): pp. 461-467.

9. Nazrul H. ADNAN et al., Principal Component Analysis For The Classification Of Fingers Movement Data Using DataGlove "GloveMAP". International Journal of Computer Engineering \& Technology (IJCET), 4(2): pp. 79-93.

10. Nazrul H. ADNAN et al., PCA-based Finger Movement and Grasping Classification using DataGlove "GloveMAP". International Journal of Innovative Technology and Exploring Engineering (IJITEE), 2(3): pp. 66-71.

11. Nazrul H. ADNAN et al., Classification Of Finger Grasping By Using PCA Based On Best Matching Unit 
(BMU) Approach. International Journal of Advanced Research In Engineering And Technology (IJARET), 4(2): pp. 92-105.

12. A. A. Ali and A. H. Miry, Human Arm Inverse Kinematic Solution Based Geometric Relations and Optimization Algorithm. IJRA, 2(4): pp. 245. 